\title{
Density waves in atomic necklaces
}

\author{
Paul C. Snijders ${ }^{1}$, Sven Rogge ${ }^{1}$, and Hanno H. Weitering ${ }^{2,3}$ \\ ${ }^{1}$ Kavli Institute of NanoScience, Delft University of Technology • 2628 CJ Delft • The Netherlands \\ ${ }^{2}$ Department of Physics and Astronomy, The University of Tennessee • Knoxville, TN $37996 \cdot$ USA. \\ ${ }^{3}$ Materials Science and Technology Division, Oak Ridge National Laboratory• Oak Ridge, TN 37831 • USA.
}

$\mathrm{O}$ ne-dimensional (1D) systems have always captured the imagination of physicists. In the ultimate $1 \mathrm{D}$ limit, a crystal is nothing but a string of atoms, akin to a necklace of tiny pearls. Most physics graduates undoubtedly associate $1 \mathrm{D}$ systems with the simple models used in introductory level quantum mechanics or statistical mechanics courses. Indeed, 1D model systems represent pedagogical and often most useful illustrations of the basic physics principles that govern more realistic condensed matter systems. These principles tell us, for instance, that an ideal atomic chain with a single metallic band should be unstable with respect to a Peierls distortion. A Peierls distortion is a condensed state of the $1 \mathrm{D}$ electron gas whose formation is triggered by the strong coupling between electrons and quantized lattice vibrations (phonons). The upshot is that such a wire would not conduct electricity at low temperature. Alternatively, atom wires could display exotic many-body physics. It has been predicted that, due to strong electron-electron interactions in $1 \mathrm{D}$, the concept of single electrons should even break down and that it is only meaningful to discuss their collective behavior (i.e., excitations). Such a state of matter is referred to as the Luttinger-Tomonaga liquid.

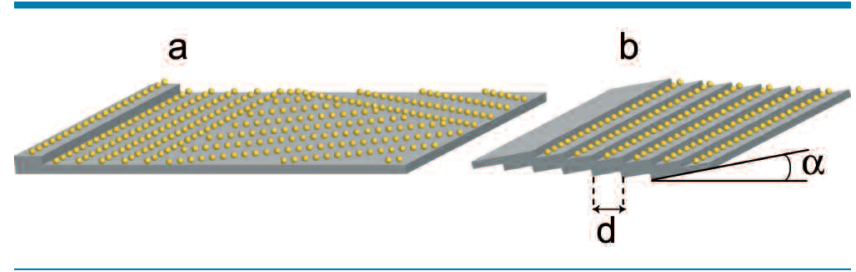

$\Delta$ Fig. 1: (a) Schematic illustration of the threefold degeneracy of anisotropic reconstructions on the Si(111) surface. Note the limited "wire-length" due to the small domain sizes. (b) The array of step edges on a vicinal $\mathrm{Si}(111)$ surface produces a single domain atomwire array. $\alpha$ and $d$ are the miscut angle and the resulting atom wire spacing, respectively.

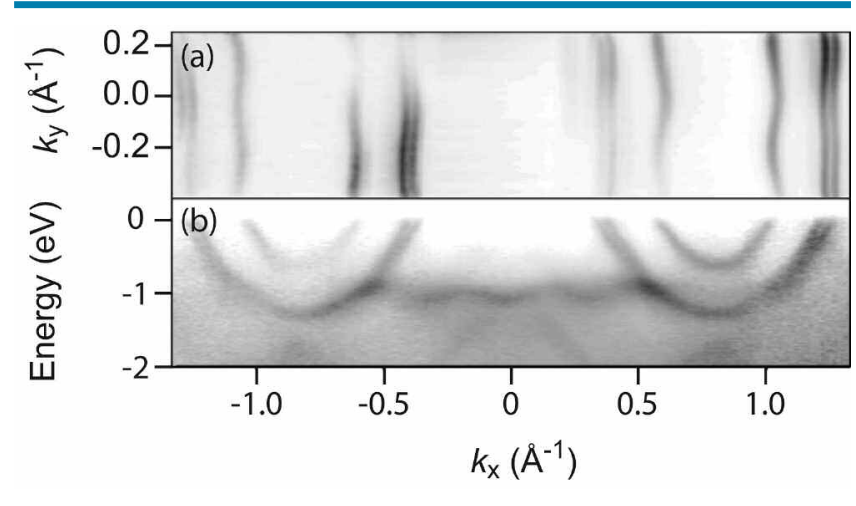

$\Delta$ Fig. 2: The Fermi surface (a) and band structure (b) of the Si(553)Au surface as measured with ARPES: photoemission intensity (dark) as a function of energy and wave vector along the wires (a), and as a function of wave vector parallel and perpendicular to the wires (b). Reprinted figure (a) and (b) with permission from Ref. [8], copyright (2003) by the American Physical Society.
But even though the theory of ideal 1D systems has advanced much, experimental realization of truly 1D condensed matter systems remains a daunting task; it is impossible to suspend a long string of atoms in free space. Nonetheless, due to the progress in nanoscience, it is now possible to synthesize and tailor novel nanophase materials that mimic the ultimate $1 \mathrm{D}$ limit quite closely. As a result, crucial questions regarding the physics of atom wires can be experimentally addressed. For instance: would a string of atoms conduct if it is laid down on a substrate? How can the often detrimental effects of the inevitable structural imperfections or those of thermodynamic fluctuations be alleviated? Or perhaps the most fundamental and practical issue: would atom wires actually be thermodynamically stable?

Experimental approaches toward creating 1D systems are often classified as 'top down' or 'bottom up' fabrication. The top-down procedure refers to artificial (lithographic) creation of $1 \mathrm{D}$ nanostructures. A natural way to produce atom wires is a bottom-up method that employs the most common 1D line-defects on surfaces, namely atomic step-edges, as a template for self-assembly. Moreover, artificially created nanostructures are generally unstable, whereas self-assembled (nano-) structures instead can be thermodynamically stable, just because the driving force in their formation is not a kinetically enforced low-dimensional ordering, but purely a drive toward the lowest energy state $[1,2]$.

We use the symmetry breaking properties of surfaces and the reconstructions of their topmost atom layers to realize self-assembled macroscopic arrays of atom wires. To this end, we have used vicinal (stepped) Si surfaces. Besides the thermodynamic stability inherent to self-assembly based fabrication, an extra advantage of this approach is that the spatial access to these atom wire arrays allows for a detailed investigation of electronic instabilities in these wires, even at a local scale. The atom wires can reach macroscopic lengths on vicinal surfaces; their length is only limited by occasional step edges crossing the wires. On the other hand, producing atom wires via this bottom-up method inevitably introduces (intrinsic) defects. This should not necessarily be viewed as a drawback, since it provides a much-needed opportunity to study the dramatic influence of imperfections in atom wires on their (in-)stabilities. Because they are located at surfaces, the wires can be studied conveniently using well-developed techniques such as Scanning Tunneling Microscopy and Spectroscopy (STM and STS), and Angle Resolved Photoemission Spectroscopy (ARPES).

\section{Atom wires on stepped Si surfaces}

The Si(111) surface displays a threefold symmetry. Consequently reconstructions with a lower symmetry will form in three degenerate domains of limited size on the surface, as illustrated in Figure 1(a) [3]. Upon adsorption of less than a monolayer of Au on a vicinal (stepped) surface, the additional anisotropy imposed by the step-edges drives the formation of a regular array of parallel 1D atom wires that can have macroscopic lengths, see Figure 1(b). Changing the miscut angle $\alpha$ between the surface plane and the (111) plane, permits a systematic study of the electronic properties as a function of inter-wire distance $d$ [3]. Note that even though 
these atom wires are mechanically supported by a bulk Si substrate, their electronic structure is decoupled from that of the bulk Si substrate because the metallic states of the atom wires lie in the band gap of the Si substrate.

\section{Electronic instabilities}

Several studies on atom wire arrays on Si surfaces have been reported in the literature so far. In particular, photoemission measurements (probing the band structure of the surface region of a solid in $k$-space) on the $\mathrm{Si}(111) 4 \times 1$-In and the $\mathrm{Si}(557)$-Au atom wire reconstructions reveal metallic bands which appear to cross the Fermi level at room temperature [4,5]. However, STM images at low temperatures reveal a doubling of the periodicity of the atom wire structure and a gap in the band structure $[4,6]$. These effects have been interpreted as evidence for the occurrence of a charge density wave or Peierls distortion (for a review on charge density waves, see Ref. [7]). A charge density wave originates from the interaction between electrons and phonons. It is usually accompanied by a structural modulation: when the band filling of the undistorted (high-symmetry) phase is $1 / n$, the lattice assumes an $n$-tupled superperiod below the transition temperature. This charge density wave is furthermore accompanied by a band-gap opening. The gap opening stabilizes the charge density wave state, in spite of the increase in lattice strain. Charge density waves are not necessarily commensurate ( $n$ does not need to be integer), but commensurate charge density waves are intuitively expected to be more stable than incommensurate charge density waves because the electronic system can more easily adapt to the periodic potential of the lattice. The gap opening possibly prevents the observation of low-energy (electron-) interaction effects close to the Fermi level at low temperatures. This means that the electrons in an atom wire may prefer to order in space, and in doing so the metallicity of the wires is destroyed.

There might be a way to prevent charge density wave ordering and the associated destruction of the metallic state. In a multi-band $1 \mathrm{D}$ system, there could be a competition between different charge density wave states and it is conceivable that the condensation of one charge density wave and accompanying lattice distortion would alleviate or even eliminate electronic instabilities in the other bands, which would then retain their metallic character. Moreover, the Coulomb interaction that is present between charge density waves with different periodicities could also inhibit the condensation of (one of these) charge density waves.
In this respect, the atom wires present on vicinal $\mathrm{Si}(553)$-Au surface show a promising electronic structure; room temperature photoemission measurements reveal three bands crossing the Fermi level, see Figure $2[3,8]$. The band fillings $1 / n$ of the three bands are $0.27,0.51$, and $0.56 \pm 0.01$, respectively $[3,8]$. This system thus constitutes a multi-band system, where at least two of the three bands (the 0.27 - and the 0.56 -filled bands) are incommensurate.

\section{Atom wires on Si(553)-Au}

In this article we discuss recent experiments on atom wires on the vicinal $\mathrm{Si}(553)$ surface [9]. The atom wires were created by depositing a submonolayer amount of $\mathrm{Au}$ onto the $\mathrm{Si}$ (553) surface. A subsequent thermal anneal at $1120 \mathrm{~K}$ ensures that the surface has reached an ordered equilibrium structure consisting of atom wires. This surface preparation takes place in an ultra high vacuum (UHV, base pressure $<5 \times 10^{-11}$ mbar) system, to protect the atom wires from ambient conditions.

Following the preparation of the atom wire arrays, the sample was transferred to the STM, which is located in the same UHV system. We have recorded STM images and STS $I-V$ curves measured at room temperature and various low temperatures [9]. In an STM, an atomically sharp tip is brought in tunneling contact with the surface, and is subsequently scanned over the surface while maintaining a constant tunneling current using a feedback loop. Due to differences in height and in the local density of states of the surface, the absolute vertical tip-position will vary so as to maintain a constant tunneling current. By plotting the change in vertical tip-position as a function of $x$ and $y$ coordinates, an atomic-scale real space image of the surface structure is obtained. Note that this image contains information on both the atomic structure and the local density of states. In Figure 3(a) we present a filled state (electrons tunneling from the filled density of states of the sample to the tip) room temperature STM image of the Si(553)-Au surface. The image shows rather smooth atom wires that are cut by seemingly random vacancy-like defects. The empty state image (not shown) is very similar. The corresponding STS $I-V$ curve is displayed in Figure 3(e). In an STS experiment we ramp the voltage between sample and tip and measure locally the tunnel current at a constant tip-sample distance. The room temperature $I$ - $V$ curve shows a finite slope at zero bias, indicating a finite density of states at the Fermi level, which is consistent with the metallic band structure measured by ARPES. However, after decreasing the
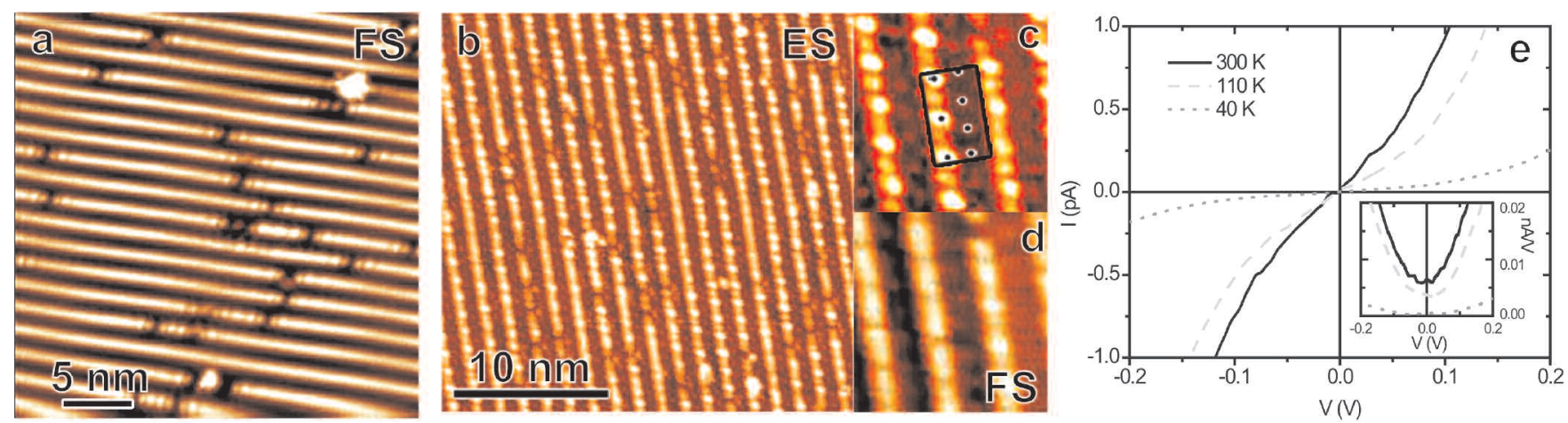

A Fig. 3: (a) Room temperature filled state (-0.5 V, 50 pA) STM image showing atom wires, locally cut by vacancy-like defects. (b) Empty state $(1 \mathrm{~V}, 100$ pA) STM image at $40 \mathrm{~K}$ and magnification (c). In (c) the tripled periodicity on the chains and doubled periodicity in between the chains are indicated. (d) Filled state (-1 V, 100 pA) STM image of the same area as (c). (e) STS I-V curves, measured at the indicated temperatures. The inset shows the numerical derivatives. Reprinted figures (a) to (e) with permission from Ref. [9], copyright (2006) by the American Physical Society. 
sample temperature to $\sim 40 \mathrm{~K}$, the measurements reveal different characteristics, see Figure 3. First of all, a clear corrugation has developed in the empty state images (Figure 3(b) and (c)). The wavelength of this corrugation is exactly equal to three times the substrate lattice constant a. In the filled state images (Figure 3(d)), the atom wires appear slightly depressed at locations where the empty state image showed enhanced intensity. This contrast reversal with changing tunnel bias polarity is the hallmark of a charge density wave. The charge density is proportional to the density of filled states, and thus the total charge density exhibits a periodic modulation; a charge density wave. Note that the wavelength of this charge density wave is commensurate, with $n=3$, even though the band fillings at high temperature clearly deviate from $1 / 3$ according to the photoemission measurements. The local $I-V$ measurements confirm the existence of a charge density wave: the $40 \mathrm{~K} \mathrm{STS} \mathrm{I-V} \mathrm{curve} \mathrm{in} \mathrm{Figure} \mathrm{3(e)} \mathrm{no} \mathrm{longer} \mathrm{exhibits} \mathrm{a} \mathrm{slope} \mathrm{around}$ zero bias indicating the presence of a band gap. From this STS measurement we can conclude that all three bands exhibit a gap and have lost their metallicity; the surface is completely semiconducting. This is confirmed by the observation of an additional $x 2$ superperiod in between the atom wires with tripled periodicity, see Figure 3(c). The new periodicity is again commensurate with the lattice $(n=2)$. Evidently, two different commensurate charge density waves coexist. They run parallel to the atom rows and are spaced less than one nm apart.

To investigate how these two commensurate charge density waves develop from an electronic structure with three incommensurate Fermi wave vectors, we have imaged the surface with STM at intermediate temperatures. At $70 \mathrm{~K}$ the tripled corrugation is vaguely visible, with significantly enhanced intensity close to defects (not shown, cf. Figure 4 in Ref. [9]). In Figure 4(a), part of an atom wire close to a defect as imaged with the STM at $110 \mathrm{~K}$ is represented as a three-dimensional contour. The corrugation of the atom wire exhibits a clear tripled periodicity close to the defect, whereas farther away from the defect, a double-period corrugation is visible. STS data at $110 \mathrm{~K}$ reveal a finite slope at zero bias, but the slope is significantly smaller than that at room temperature, see Figure 3(e). The reduced density of states at the Fermi level at 110 $\mathrm{K}$ is consistent with a double-period charge density wave in one of the bands, while the other two bands remain metallic. From the $110 \mathrm{~K}, 70 \mathrm{~K}$, and $40 \mathrm{~K} \mathrm{STM}$ data, it therefore appears that the tripleperiod charge density is defect mediated, i.e. it nucleates at the defects, and grows outward into the bulk of the chain at lower temperatures. However, the doubled periodicity in the chains visible at $110 \mathrm{~K}$ is a much more interesting observation. First, it is clear that a competition exists between two different charge density wave orders within a single atom wire. These two charge density waves, having different transition temperatures but competing at $110 \mathrm{~K}$, provide the first observation of two competing charge density wave orders in a single atom wire. For example, (bulk) $\mathrm{NbSe}_{3}$ shows two consecutive charge density wave transitions, but those are localized on different "chains" within the unit cell and, consequently, they will have a fundamentally different and much smaller interaction. Secondly, the observation of this second charge density wave within the atom wire allows for a consistent explanation of the low and intermediate temperature electronic structure of a triple-band atom wire system. This explanation will be given in the following Section.

\section{The role of defects}

Summarizing the STM and STS observations provides the following data: at $40 \mathrm{~K}$ we observe a triple-period charge density wave on the atom wires and a double-period charge density wave between these wires, and at $110 \mathrm{~K}$ we observe an additional doubleperiod charge density wave on the wires, all commensurate with the substrate lattice. Photoemission measurements (Ref. [3,8]) reveal three bands, that are 0.27-, 0.51-, and 0.56-filled. The Fermi wave vectors are all incommensurate with the chain lattice. We invoke an interband charge transfer of 0.06 from the 0.56 -filled band to the 0.27 -filled band to explain these commensurate periodicities. This results in exactly commensurate $1 / 2$-filled and $1 / 3$-filled bands, naturally explaining the observed triple period charge density wave in the atom wires and the double period charge density wave between them. Keeping in mind that these charge density waves appear to nucleate at the defects, we can conclude that these defects act as dopants in the atom wires; they change the band-filling, and thus mediate the formation of these two charge density waves. (Our placement of the 0.56 -filled band between the atom wires is corroborated by the simultaneous room temperature observation of a local tripled and doubled corrugation on and in between the atom wires very close to the defects, respectively, cf. the discussion and References in Ref. [9].) This leaves the 0.51 -filled band responsible for the double period charge density wave that evidently competes with the triple period charge density wave at $110 \mathrm{~K}$.

Apparently, the defects present in the atom wires do not simply disrupt or destabilize the atom wire system. Instead, they appear to stabilize the system via a doping mechanism that ultimately favors the commensurate charge density wave state. The energy gain even outweighs a likely strain-energy cost due to the incorporation of these defects.
Fig. 4: (a) 3D representation of an

atom wire next to a defect. Close to the defect a tripled periodicity is visible (blue arrows), whereas farther away from the defect a doubled periodicity appears (red arrows).

(b) 3D representation of an atom wire. A clear tripled periodicity is visible, with at one location a phase slip of $2 \pi / 3$, i.e. the distance between two maxima is $4 a$ instead of $3 a$. As indicated schematically, in the periodic charge density wave, each unit cell (black arrow) contains two electrons, but an extra fractional charge and spin is located at the kink (red arrow).

Note that here the case for a singly occupied kink-state is shown; the actual fractional quantum numbers depend on the filling of the kink-state [9]. a

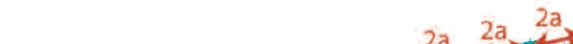

$2 a 2 a 2 a$

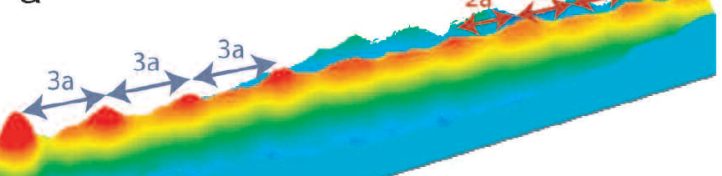

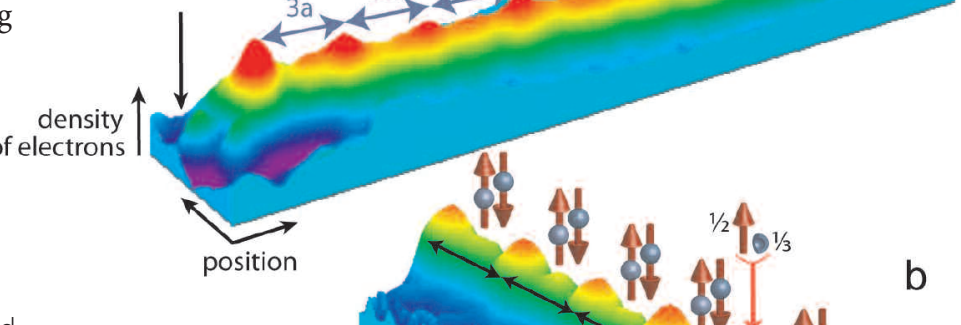




\section{Fractional charges}

In our experiments at $40 \mathrm{~K}$ we observed several phase slips in the triple-period charge density wave, as demonstrated in Figure 4(b). These phase slips or solitons show a jump of $2 \pi / 3$ in the phase of the triple period charge density wave. It is interesting to note that Schrieffer calculated that such phase slips should have fractional (spin,charge): either $(1 / 2, \pm e / 3)$ or $(0, \pm 2 e / 3)$, depending on the filling of the localized kink-state [10]. Fractional quantum numbers (with the exception of the spin quantum number) only occur in excitations of a collective ground state. A famous example is given by the fractional quantum Hall effect, displayed by a $2 \mathrm{D}$ electron gas in high magnetic fields. Here an integer number $m$ of magnetic flux quanta (vortices) is attached to each electron. When the magnetic field slightly exceeds the integer number $m$ of flux quanta per electron, the system is excited by the excess magnetic field and single magnetic flux quanta are "left over". The deficit of charge in these vortices amounts to the fractional charge $e / m$ [11]. Thus, excitations of a collective system of electrons and magnetic flux quanta can produce fractional quantum numbers. Equivalently, the observed solitons represent excited states of the collective (electron and phonon) triple-period charge density wave ground state. Fractional charges have been experimentally studied mostly using electrical transport (in the case of the fractional quantum Hall effect) or with optical methods (for bulk charge density wave systems). Such experiments are inherently (bulk-)averaging; signatures of fractional charges can be deduced, but individual solitons are not observed. For the first time, these fractional charges are experimentally accessible through individual solitons in atom wires. STM and STS thus allow for a real space study of the electronic structure of these solitons at the atomic scale.

In this regard, the defects in the atom wires again might play an important role: Figure 5 shows two sequential ( $\Delta t \sim 180 \mathrm{~s}$ ) filled state STM images taken at $40 \mathrm{~K}$. The black circles serve as markers. It is clear that in between the two scans, the configuration of defects indicated by the arrow has changed. A close inspection of another defect in the same atom wire (inside the black rectangle) even shows that this defect is generated when the tip is scanning the atom wire; the defect seems to be present only in the right half of the atom wire. Even though this rearrangement of defects was not intentional, these observations indicate that it is possible to move these defects with an STM tip. It might thus be possible to locate two defects in a single atom wire, separated by for example ten lattice constants. This will induce a phase slip in the triple-period charge density wave, allowing for a systematic study of the electronic properties of these phase slips in preselected locations.

In conclusion, the example provided by the $\mathrm{Si}(553)$-Au surface, nicely illustrates the role of defects in the electronic structure of self-assembled $1 \mathrm{D}$ atom wires. They play a pivotal role in the thermodynamic stabilization of the atom wires, and furthermore provide possibilities to engineer specific structures to study the electronic structure of 1D atom wires. Scanning Tunneling Microscopy and Spectroscopy provides a much needed opportunity to determine the precise role of defects in fluctuations and stabilization of $1 \mathrm{D}$ atom wires.

\section{About the authors}

Paul C. Snijders received his Masters Degree in Materials Science at the Delft University of Technology, and investigated these atomic wire systems for his Ph.D. research at the Kavli Institute of NanoScience in Delft.

Sven Rogge received his Ph.D. in Physics from Stanford University in 1997. He works on atomic-scale electronics in semiconductors at the Kavli Institute of NanoScience Delft where he holds a faculty position.

Hanno H. Weitering received his Ph.D. in the physical sciences at the University of Groningen. He has been working in the areas of surface physics and nanoscience and holds a Joint-Faculty Chair of Excellence at The University of Tennessee and Oak Ridge National Laboratory.

\section{References}

[1] C. González, P.C. Snijders, J. Ortega, R. Pérez, F. Flores, S. Rogge and H.H. Weitering, Phys. Rev. Lett. 93, 126106 (2004).

[2] P.C. Snijders, S. Rogge, C. González, J. Ortega, R. Pérez, F. Flores, and H.H. Weitering, Phys. Rev. B 72, 125343 (2005).

[3] J.N. Crain, J.L. McChesney, F. Zheng, M.C. Gallagher, P.C. Snijders, M. Bissen, C. Gundelach, S.C. Erwin and F.J. Himpsel, Phys. Rev. B 69, 125401 (2004).

[4] H.W. Yeom, S. Takeda, E. Rotenberg, I. Matsuda, K. Horikoshi, J. Schaefer, C.M. Lee, S.D. Kevan, T. Ohta, T. Nagao, and S. Hasegawa, Phys. Rev. Lett. 82, 4898 (1999).

[5] R. Losio, K.N. Altmann, A. Kirakosian, J.-L. Lin, D. Petrovykh and F.J. Himpsel, Phys. Rev. Lett. 86, 4632 (2001).

[6] J.R. Ahn, H.W. Yeom, H.S. Yoon and I.-W. Lyo, Phys. Rev. Lett. 91, 196403 (2003).

[7] G. Grüner, Rev. Mod. Phys. 60, 1129 (1988).

[8] J.N. Crain, A. Kirakosian, K.N. Altmann, C. Bromberger, S.C. Erwin, J.L. McChesney, J.-L. Lin and F.J. Himpsel, Phys. Rev. Lett. 92, 176805 (2003), see also Phys. Rev. Lett. 92, 089902(E) (2004).

[9] P.C. Snijders, S. Rogge and H.H. Weitering, Phys. Rev. Lett. 96, 076801 (2005).

[10] W.P. Su and J.R. Schrieffer, Phys. Rev. Lett. 46, 738 (1981).

[11] H.L. Stormer, Rev. Mod. Phys. 71, 875 (1999).

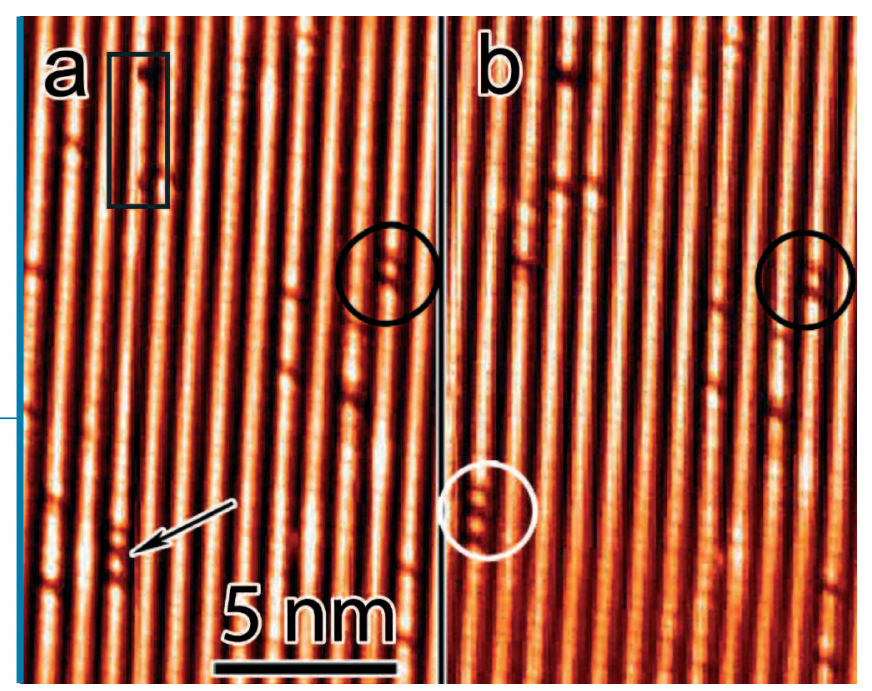

